\title{
Oscillatory power decreases and long-term memory: the information via desynchronization hypothesis
}

\author{
Simon Hanslmayr ${ }^{1,2 *}$, Tobias Staudigl ${ }^{1}$ and Marie-Christin Fellner ${ }^{1}$ \\ Department of Psychology, University of Konstanz, Konstanz, Germany \\ ${ }^{2}$ Zukunftskolleg, University of Konstanz, Konstanz, Germany
}

Edited by:

Simone Rossi, Azienda Ospedaliera

Universitaria Senese, Italy

\section{Reviewed by:}

Fernando Maestú, Complutense University, Spain

Leun J. Otten, University College

London, UK

*Correspondence:

Simon Hanslmayr, Department of Psychology, University of Konstanz,

Box 23/25, 78457 Konstanz,

Germany.

e-mail: simon.hanslmayr@

uni-konstanz.de
The traditional belief is that brain oscillations are important for human long-term memory, because they induce synchronized firing between cell assemblies which shapes synaptic plasticity. Therefore, most prior studies focused on the role of synchronization for episodic memory, as reflected in theta $(\sim 5 \mathrm{~Hz})$ and gamma $(>40 \mathrm{~Hz})$ power increases. These studies, however, neglect the role that is played by neural desynchronization, which is usually reflected in power decreases in the alpha and beta frequency band $(8-30 \mathrm{~Hz})$. In this paper we present a first idea, derived from information theory that gives a mechanistic explanation of how neural desynchronization aids human memory encoding and retrieval. Thereby we will review current studies investigating the role of alpha and beta power decreases during long-term memory tasks and show that alpha and beta power decreases play an important and active role for human memory. Applying mathematical models of information theory, we demonstrate that neural desynchronization is positively related to the richness of information represented in the brain, thereby enabling encoding and retrieval of long-term memories. This information via desynchronization hypothesis makes several predictions, which can be tested in future experiments.

Keywords: oscillations, desynchronization, synchronization, long-term memory, EEG, MEG, alpha, beta

\section{INTRODUCTION}

One of the most influential ideas in memory research has been that memories are stored in the synaptic weights of neural assemblies (Hebb, 2002), which are shaped by synchronized activity (Markram et al., 1997). Brain oscillations refer to the regular fluctuations in the local field potential, reflecting the input of thousands of neurons to a specific cell assembly. Thereby, brain oscillations index graded excitatory or inhibitory postsynaptic potentials, which are picked up by EEG/MEG sensors. These synchronous fluctuations between excitation and inhibition have been shown to induce synchronized firing patterns (Lee et al., 2005; Jacobs et al., 2007; Haegens et al., 2011). Brain oscillations are, therefore, considered to be one of the core neural mechanisms for the storage and retrieval of long-term memories (Buzsáki and Draguhn, 2004; Fell and Axmacher, 2011). Indeed, several studies using intracranial EEG, surface EEG, and MEG have shown that brain oscillations play a crucial role for long-term memory (see Axmacher et al., 2006; Düzel et al., 2010; Nyhus and Curran, 2010, for recent reviews). The overriding theme in these studies is that increases in synchronized activity in the theta (around $5 \mathrm{~Hz}$ ) and gamma $(>40 \mathrm{~Hz})$ frequency ranges play an important role for memory formation and retrieval via shaping synaptic plasticity and coordinating the reactivation of memories.

A less attended fact, however, is that decreases in synchrony also strongly correlate with the formation and retrieval of longterm memories (Figure 1). Such desynchronization is typically observed in the alpha $(8-12 \mathrm{~Hz})$ and beta band $(13-35 \mathrm{~Hz})$ and is reflected by a reduction of post-stimulus power compared to a pre-stimulus baseline period (Pfurtscheller and Aranibar, 1977). Note, however, that the exact limits for these frequency bands may vary from study to study. Although various studies demonstrated that such relative alpha and beta power decreases can be robustly observed during long-term memory tasks, no theoretical framework yet exists to explain how such decreased synchrony in neural assemblies could serve the encoding and retrieval of memories. This review is a first step in this direction and proposes a mechanistic idea that links decreased synchrony to concepts derived from information theory (e.g., entropy). Before laying out this idea we will start with a current review of empirical studies investigating alpha and beta power decreases during memory encoding (Section "Alpha and Beta Power Decrease Correlates with Successful Memory Encoding") and memory retrieval (Section "Alpha and Beta Power Decrease Correlates with Memory Retrieval"). Thereafter, we will discuss the neurophysiological underpinnings of alpha and beta power decreases and demonstrate that these power decreases are not an epiphenomenon of increases in higher frequency ranges (Section "Neurophysiological Considerations of Alpha/Beta Power Decreases"). In Section "Neural Desynchronization, Memory and Information Theory", the information via desynchronization hypothesis is presented suggesting that desynchronization is a prerequisite for information encoding and showing how desynchronized activity could induce long-term potentiation (LTP) in neural assemblies. Section "Relation to Other Theories" briefly describes the relation of the presented framework to other, relevant concepts in the field. Finally, open questions and testable 
predictions that follow from this idea will be discussed at the end of the paper (Section "Concluding Remarks, Testable Predictions, and Open Questions").

In this review, we will focus on studies investigating explicit long-term memory, which required participants to retrieve memory items that were presented in a previous list, with a distracter task in between. Thereby, most of the tasks in the reviewed studies would fall into the domain of episodic memory, as defined by Tulving (1972).

“... Consider now a typical memory experiment in which a subject is asked to study and remember a list of familiar words or pairs of words. This is an episodic memory task. The occurrence of a verbal item in a given list, at a particular time, and in specified temporal relation to other items in the list is an autobiographical episode having no necessary extra-episodic denotative reference (Tulving, 1972, pp. 393)."

However, as episodic memory has also been strongly linked to the availability of contextual information accompanying the

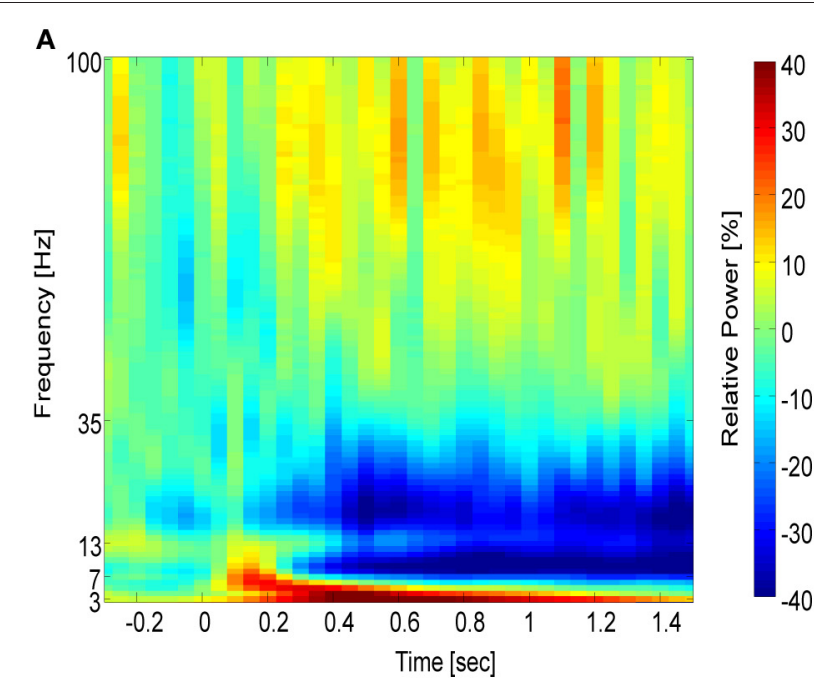

B

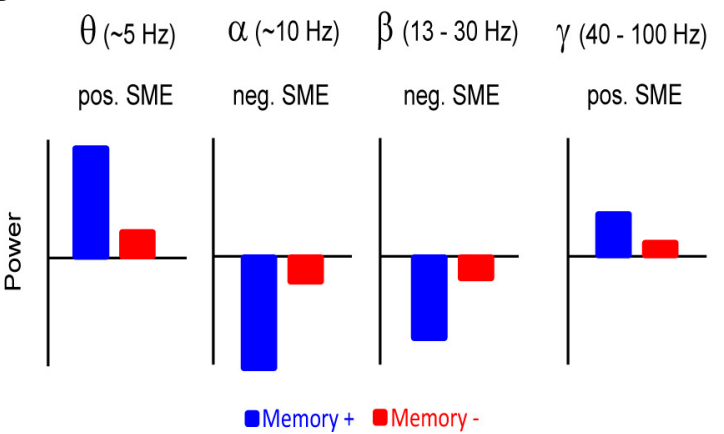

FIGURE 1 | The different frequency bands and their relation to memory. (A) A time-frequency power spectrum of a parietal electrode (Pz) is shown during a memory task. Time 0 indicates stimulus presentation. Power increases relative to baseline (red) can be seen in the theta and gamma frequency range, whereas relative power decreases (blue) emerge in the alpha and beta frequency range. (B) A schematic depiction of the power in the different frequency bands is shown and how they typically relate to memory. Blue refers to items for which memory succeeds (Memory+), red refers to items for which memory fails (Memory-). study event, the more neutral term long-term memory will be used as most of the reviewed studies did not explicitly test for such context information.

\section{ALPHA AND BETA POWER DECREASE CORRELATES WITH SUCCESSFUL MEMORY ENCODING}

In this section, we will mostly focus on studies investigating the so-called subsequent memory (SM), or difference in memory (DM) paradigm. In this paradigm, neural activity during encoding is contrasted based on memory performance in a later test. For example, the neural activity elicited by subsequently remembered items (words, pictures, etc.) is compared with neural activity to items that are subsequently forgotten (Paller and Wagner, 2002) (see Figure 2A). Activity in brain regions or brain oscillatory activities differentiating between retrievable and non-retrievable

A $\alpha / \beta$ Power during encoding

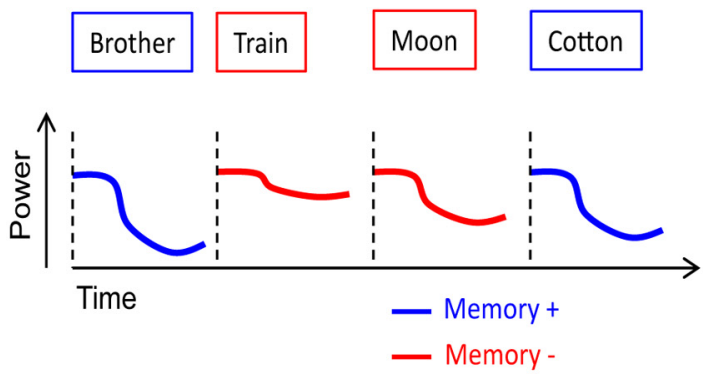

B $\alpha$ - Power $\beta$ - Power
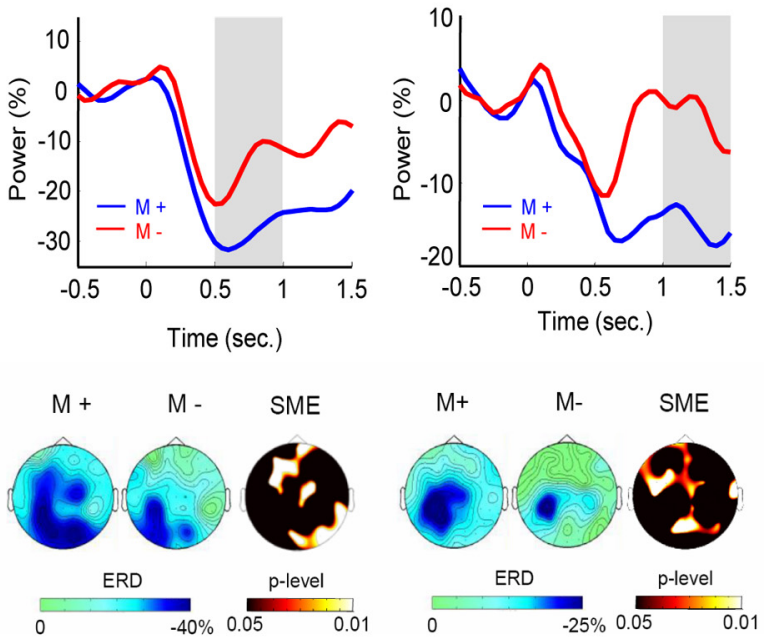

FIGURE 2 | Alpha/beta power during encoding. (A) Alpha/beta power decreases during encoding are more pronounced for words which are subsequently remembered (blue) than for words which are subsequently forgotten (red). (B) Alpha (left) and beta (right) SMEs are shown during deep (semantic) encoding. Negative SMEs were observed in both frequency bands from 500 to $1500 \mathrm{~ms}$ after stimulus onset. The gray bars indicate the time windows for plotting the corresponding topographies (bottom). Figure adapted from Hanslmayr et al. (2009a). 
items are referred to as subsequent memory effects (SMEs). Thereby, the neural activity during encoding can positively or negatively correlate with later retrieval, termed positive or negative SMEs (Figure 1B). Whereas positive SMEs, i.e., increases in power for subsequently remembered items, are usually found in the theta and gamma frequency ranges (see Düzel et al., 2010; Nyhus and Curran, 2010; for reviews), negative SMEs, i.e., decreases in power for subsequently remembered items have been reported in the alpha and beta frequency range (e.g., Klimesch et al., 1996; Sederberg et al., 2003; Hanslmayr et al., 2009a).

Among the first researchers noticing negative SMEs in alpha power was Klimesch, who showed that decreases in parietal alpha power during semantic encoding are positively related to later retrieval (Klimesch et al., 1996, 1997). In these studies, Klimesch et al. divided the alpha band into a lower $(\sim 8-10 \mathrm{~Hz})$ and an upper frequency band $(\sim 10-12 \mathrm{~Hz})$ based on the individual dominant frequency of the subjects. The findings led Klimesch to conclude that the upper alpha band specifically reflects semantic memory processes (Klimesch, 1999). In these early studies beta power was not analyzed, presumably because beta oscillations were predominantly linked to motor activity, rather than memory processes (Pfurtscheller et al., 1997). Several EEG studies followed which replicated these negative SMEs in the alpha band, demonstrating the robustness of this effect (Weiss and Rappelsberger, 2000; Sederberg et al., 2006; Hanslmayr et al., 2009a).

With increasing computational power, it became feasible to analyze the whole frequency range and recent EEG studies found not only negative SMEs in the alpha, but also in the adjacent beta frequency range $(\sim 15 \mathrm{~Hz})$. These beta SMEs showed a very similar behavior as the negative SMEs in the alpha band. For instance, investigating the effect of serial positions on later memory, Sederberg et al. (2006) demonstrated negative SMEs in the alpha/beta frequency range for items which were presented in middle positions of a list. In all of the prior studies, however, the encoding strategies were either held constant (e.g., semantic as in the studies by Klimesch), or left uncontrolled (e.g., intentional encoding). We, therefore, investigated whether the brain oscillatory SMEs differ between specific encoding strategies. Such an approach should shed more light onto the cognitive processes underlying the negative brain oscillatory SMEs. In this study (Hanslmayr et al., 2009a), a semantic (deep) encoding condition was contrasted with a non-semantic (shallow) encoding condition. The results showed that the negative SMEs in the beta band $(12-20 \mathrm{~Hz})$, and to some extent also in the alpha band $(8-12 \mathrm{~Hz})$, were exclusively related to semantic encoding (Figure 2B). These findings show that semantic processing, which is known to be a very effective encoding strategy (Craik, 2002), is most strongly accompanied by these alpha and beta power decreases. Interestingly, the beta SMEs showed a left frontal topography, which is consistent with a source in the left inferior prefrontal cortex. In line with this notion, a recent MEG study (Meeuwissen et al., 2011b) localized beta power decreases during memory formation of semantically processed words in the left inferior prefrontal cortex.

Two recent studies using EEG (Khader et al., 2010) and MEG (Meeuwissen et al., 2011a) demonstrated positive SMEs in the alpha band, that is more power for later remembered in contrast to later not remembered items, which seems to be at odds with the above reported negative SMEs in this frequency range. However, the design that was employed in these two studies differed substantially from the standard subsequent memory design used in the prior studies. In their experiments, Khader et al. (2010) and Meeuwissen et al. (2011a) utilized a combined working memory-long-term memory paradigm, in which the items were first presented and then followed by a working memory rehearsal period during which no stimuli were shown. Both studies investigated whether alpha power during this (stimulation free) rehearsal period distinguished between items that were remembered in a later long-term memory test. This design is, therefore, in stark contrast to the other studies, which investigated alpha and beta power during the interval at which the items were presented. It has recently been proposed that alpha power regulates the information flow in the cortex via inhibition of task irrelevant regions (Klimesch et al., 2007; Jensen and Mazaheri, 2010). Furthermore, it has been suggested that such a shutdown of occipito-parietal areas reflects an internally oriented processing mode (Hanslmayr et al., 2011a). These positive alpha SMEs can, therefore, be interpreted as reflecting the de-activation of task irrelevant visual processing areas during the internal maintenance of items in working memory (Jensen et al., 2002; Meeuwissen et al., 2011a). Conversely, the negative SMEs during the presentation of the memory items might reflect the activation of encoding-relevant brain regions.

In intracranial EEG studies the EEG is usually recorded in patients suffering from pharmaco-resistant epilepsy, enabling researchers to record brain oscillations during memory formation with high anatomical precision. Performing such experiments, Sederberg and colleagues reported negative SMEs in the alpha and beta frequency band (Sederberg et al., 2003, 2007). In a first study, the data of 10 patients were analyzed and, although the authors focused more on the positive SMEs in the theta and gamma frequency range, robust negative SMEs in the alpha and beta range $(9-28 \mathrm{~Hz})$ emerged across various cortical sites. Notably, the SMEs in the alpha and beta band were highly correlated (0.91) suggesting that they both reflect similar cognitive processes. In a later study, Sederberg and colleagues analyzed the data of 35 patients (Sederberg et al., 2007) and again demonstrated large negative alpha and beta SMEs. Besides other brain regions, the negative alpha and beta SMEs emerged in the hippocampus (collapsed over both hemispheres) and the left inferior prefrontal cortex (BA 47), both regions which are implicated in memory encoding, as demonstrated by several fMRI studies (Paller and Wagner, 2002; Kim, 2011). In their study, Sederberg et al. (2007) also observed negative SMEs in the hippocampus in the lower gamma frequency band $(\sim 30-40 \mathrm{~Hz})$, a finding which has also been reported by Fell et al. (2001). However, it seems plausible that these decreases in lower gamma power reflect a "bleed in" from the strong negative SMEs in the beta frequency range. Such "bleed ins" of beta activity into the gamma frequency can arise when the filter is tuned to give a high time resolution, as it is typically the case when filtering EEG/MEG data in the gamma range, which inevitably leads to a weak resolution in the frequency domain. 
Taken together, the findings from intracranial EEG studies complement the findings from the non-invasive EEG and MEG studies. Taking advantage of the high spatial resolution of intracranially recorded EEG, these studies demonstrate that the decreases in local alpha and beta synchrony occur in brain regions that are highly relevant for memory encoding, such as the medial temporal lobe and the (left) inferior prefrontal cortex.

The above described studies, suggest that beta power decreases specifically reflect semantic processing of memory items (Hanslmayr et al., 2009a), and are localized to the left inferior prefrontal cortex (Meeuwissen et al., 2011b), a brain region which is classically linked to semantic processing (Gabrieli et al., 1998) and semantic memory encoding (Otten and Rugg, 2001). It is, therefore, reasonable to hypothesize that the negative SMEs in the beta frequency range are generated in the left inferior prefrontal cortex and correlate negatively with the BOLD signal in this brain region. This hypothesis was tested in a recent simultaneous EEG-fMRI study (Hanslmayr et al., 2011b). The subjects performed several study-test cycles in which they intentionally encoded lists of words and, after a short distractor task, remembered these words in a free recall test. Investigating the brain oscillatory SMEs we replicated the previous findings, showing robust power decreases in the beta frequency range for later recalled in contrast to later not recalled items. This effect showed a left frontal topography and was localized to the left inferior prefrontal cortex (Figure 3A). In the fMRI, the strongest SMEs also appeared in the left inferior prefrontal cortex (green areas in Figure 3B). Furthermore, the BOLD signal in the left inferior prefrontal cortex correlated negatively with single trial beta power (red and yellow areas in Figure 3B). Crucially, this negative beta power-BOLD correlation was only present during encoding of words that were later recalled $(\mathrm{M}+)$, whereas no correlation emerged for later not recalled items (M-; Figure 3C).

This study is a first step to link brain oscillatory and fMRI SMEs, and demonstrates that the beta power decreases during successful memory formation are strongly linked to activity in the left inferior prefrontal cortex, which has been shown to support memory encoding in several fMRI studies (Otten and Rugg, 2001; Paller and Wagner, 2002; Kim, 2011). Therefore, the correlation between beta power decrease and the energy consumption in a highly task relevant brain region supports the interpretation of decreases in beta power being important for memory encoding. This result is in line with previous observations showing that the brain usually consumes less energy during states of high alpha and beta oscillatory activity (Laufs et al., 2003; Moosmann et al., 2003; Mantini et al., 2007).

The above cited studies strongly suggest that power decreases in the alpha and beta frequency ranges play an active role for the successful encoding of memories. There are, however, some limitations of these studies which deserve a brief consideration. First, most of the prior studies used visually presented neutral words, shown as single items in a list, as memory material (with exception of the study by Weiss and Rappelsberger, 2000; who also presented words acoustically). It is thus at the moment unclear to which extent alpha and beta power decreases can be generalized to other item material in terms of sensory modality, emotionality and associativity (e.g., item pairs instead of single items). There
A

$$
\beta-\text { SME }
$$

$(17-20 \mathrm{~Hz} ; 0-1.25 \mathrm{sec}$.
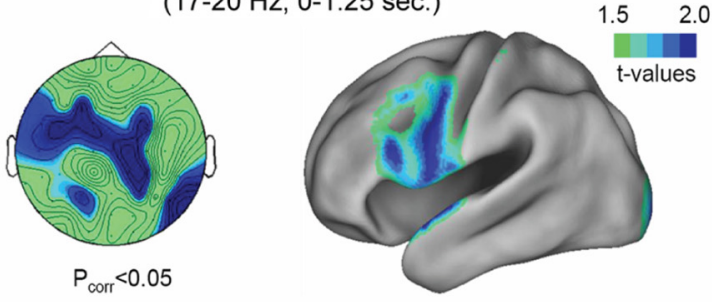

B

Correlation with BOLD

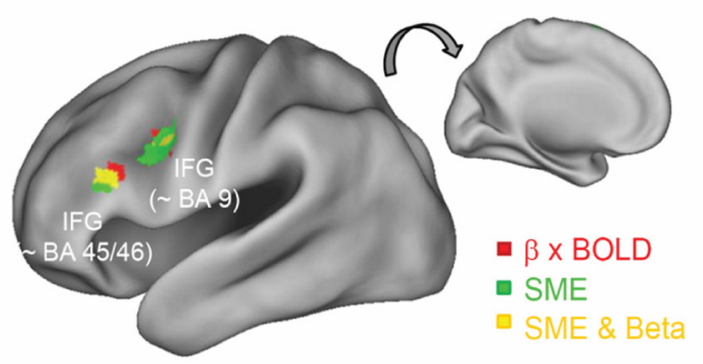

C

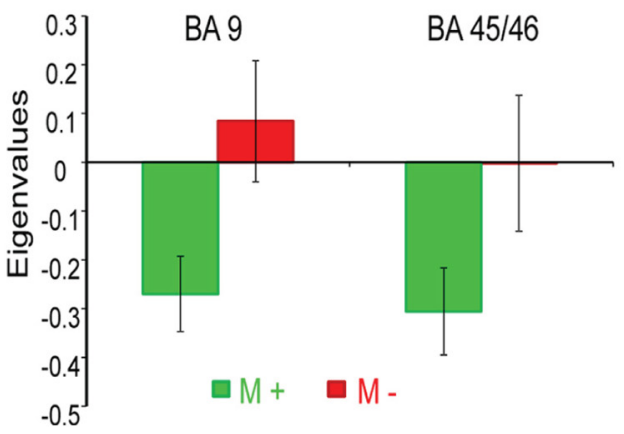

FIGURE 3 | Beta power decrease and BOLD signal during memory formation. (A) The topography of the beta SME (left) is shown together with the source localization (right). Stronger power decreases for subsequently remembered in contrast to forgotten items was observed in the left inferior frontal gyrus (IFG). (B) The negative correlation between beta power and BOLD signal is shown in red, together with the SME in the fMRI, shown in green; yellow areas denote an overlap between fMRI SMEs and negative BOLD-beta power correlations. (C) The correlation between beta power and BOLD is shown for two regions within the left IFG as a function of subsequent memory ( $\mathrm{M}+\mathrm{vs}$. $\mathrm{M}-$ ). Figure adapted from Hanslmayr et al. (2011b).

is also a lack of studies replicating and extending the brain oscillatory correlates of memory formation across different encoding strategies (Hanslmayr et al., 2009a). This is somewhat surprising given the fact that encoding strategies play a very important role in cognitive theories of memory (Tulving and Thomson, 1973; Craik, 2002) and produced a vast body of experimental findings that cognitive neuroscientists should utilize in future studies.

Second, in the above section we focused on studies investigating the brain oscillatory correlates of memory formation by analyzing stimulus-induced changes in alpha/beta power. However, 
two recent studies demonstrated that brain oscillatory activity preceding and surrounding stimulus presentation, also predicts successful formation of episodic memories (Guderian et al., 2009; Fell et al., 2011; see Addante et al., 2011; for similar results during retrieval). These studies demonstrated increased pre- and peristimulus power in medial temporal lobe regions in the theta and alpha frequency range for later remembered, in contrast to later not remembered items. These results suggest that the brain state during which an item is presented plays an important role in whether this item will be encoded or not. An interesting question for future research is whether these brain states interact with the stimulus-induced changes in alpha and beta power. For instance, does high pre-stimulus theta power correlate with a post-stimulus decrease in beta power, thereby promoting memory encoding? These are important questions which hopefully will be addressed in future studies.

As mentioned above, alpha and beta power decreases are usually measured relative to a pre-stimulus baseline. Therefore, differences in baseline activity between two conditions can lead to artificial differences in post-stimulus activity. It is, therefore, crucial to ensure that the baselines do not differ between the conditions of interest, as it was done in the studies from our laboratory (Hanslmayr et al., 2009a, 2011b).

\section{ALPHA AND BETA POWER DECREASE CORRELATES WITH MEMORY RETRIEVAL}

In this section we will review EEG and MEG studies which investigated alpha and beta power decreases during retrieval of episodic memories. Thereby, we will focus on studies which employed cued recall paradigms, or the so-called old/new recognition paradigm. In the old/new recognition paradigm, the brain oscillatory activity elicited by studied (old) items, which are correctly recognized (hits), is contrasted with activity elicited by correctly recognized new items (correct rejections; Figure 4A).

Several early EEG studies document the tight relationship between alpha and beta power decreases and memory retrieval (Dujardin et al., 1994; Klimesch, 1999; Klimesch et al., 2001). In a seminal MEG/EEG study, Düzel et al. (2003) investigated brain oscillatory responses in an old/new paradigm with visually presented words as memory material. Analyzing the entire frequency range and applying multivariate statistical analysis, the authors found that hits elicited a stronger decrease in beta power over left temporal sensors. Similar effects were also found in two recent EEG studies using words (Spitzer et al., 2009) and faces (ZionGolumbic et al., 2010) as memory items. In both studies, the decrease in alpha and beta power showed a parietal topography. It thus appears that alpha and beta power decreases do not only reflect successful memory encoding, but also successful retrieval of memories.

The crucial question is whether these power decreases can be related to a specific process during memory retrieval. In a first attempt to answer this question, Burgess and Gruzelier (2000) compared power decreases in the upper alpha, lower beta frequency range $(\sim 10-13 \mathrm{~Hz})$ during recognition of words and faces in a continuous recognition task. Their results showed that the topography of the old/new effect strongly depended on the to-beretrieved material, with words inducing stronger power decreases

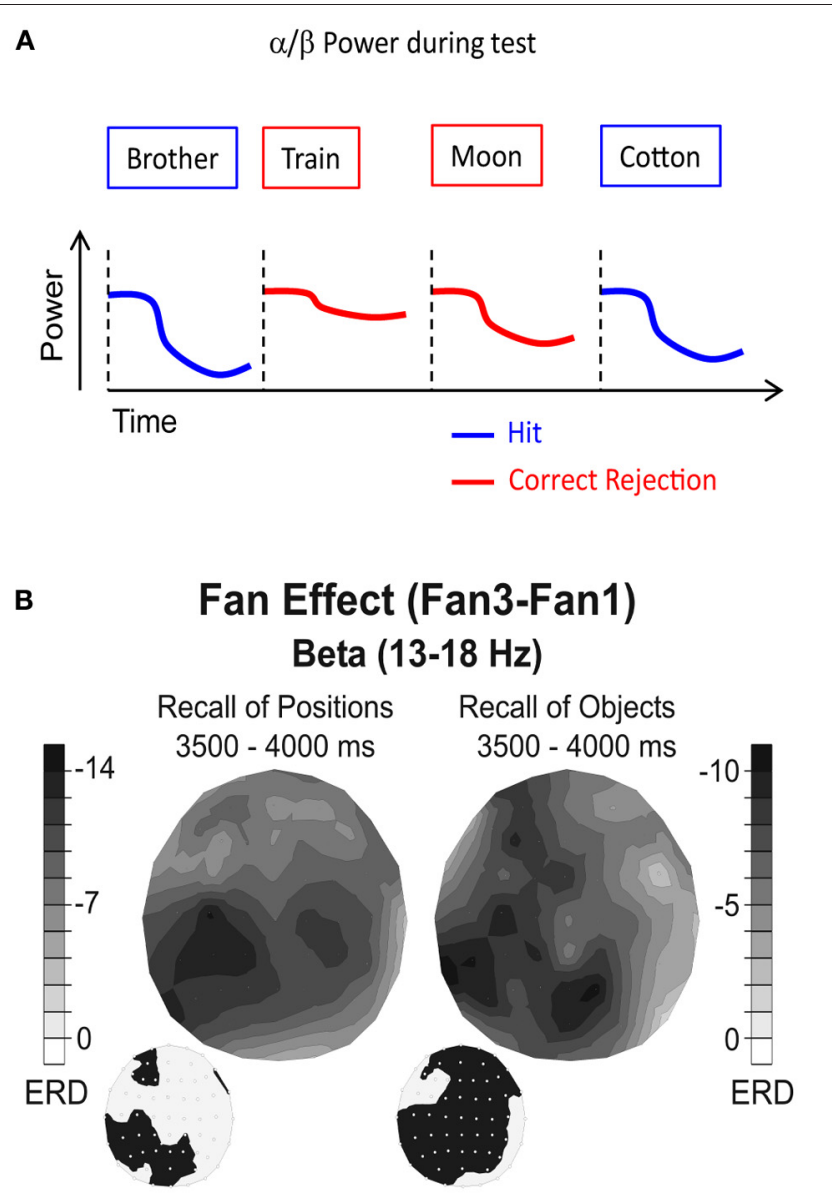

FIGURE 4 | Alpha/beta power during memory retrieval. (A) Alpha/beta power decreases are typically more pronounced during recognition of old items (hits), compared to correctly identified new items (correct rejections). (B) Beta power during retrieval of positions (left) and objects (right) is shown. Black colors denote the difference in relative power decreases (ERD) between a condition in which two items (Fan1) or four items (Fan3) were recalled. The topographies on the lower left side denote the electrode positions showing a significant difference between the two Fan conditions. Note the different topographies between recall of positions and recall of objects. Figure reproduced with permission from Khader and Rösler (2011).

over left parietal sensors, whereas faces induced stronger power decreases over right parietal sensors. The results of this study thus suggest that the alpha and beta power decreases might reflect the reactivation of the sensory features of a memory trace.

This idea was explicitly tested in a recent study by Khader and Rösler (2011). In their study, subjects were instructed to recall either objects (pictures of cups) or spatial positions (squares on a $5 \times 5$ grid), which were associated to cues (abstract nouns). Thereby, each cue was either associated with one or two items (objects or positions). During retrieval, two cue words were presented. This design allowed the authors to parametrically modulate the number of retrieved items (2,3, or 4) and the type of the retrieved material (objects or positions), while holding the sensory stimulation constant. Therefore, any material specific effects can be genuinely related to the reactivation of the sensory features 
of a memory trace. The results of this study show that alpha/beta power decreases vary systematically as a function of the number of retrieved items, with higher numbers of retrieved items inducing stronger power decreases (Figure 4B). Crucially, this fan effect also differed significantly between the types of material which had to be retrieved, such that recall of positions was reflected by an alpha and beta power decrease over parietal sites, whereas retrieval of objects was reflected by a more widespread alpha/beta power decrease over frontal, parietal, and left temporal electrode sites (Figure 4B). No such material specific reactivation effects were obtained in the theta frequency band, which varied only as a function of the numbers of retrieved items. This latter result is consistent with the idea that theta power during retrieval reflects higher order memory control processes (Hanslmayr et al., 2010; Staudigl et al., 2010).

A recent study investigated the relation between alpha/beta power decreases and the sensory reactivation of memories during selective retrieval (Waldhauser et al., 2012). In this study, subjects associated abstract shapes with color patches, whereby the shapes were presented centrally and the colors were shown in either the left or right visual field (Figure 5A). This design enabled Waldhauser et al. to link the sensory reactivation of the memory items in the left or right hemisphere (Gratton et al., 1997). Each shape was either associated with two different colors, or with only one color. In a selective retrieval task, the subjects were presented with the shape and their task was to selectively retrieve one target color, specified by a cue (Figure 5B). Presumably, retrieval in such an ambiguous condition, leads to competition elicited by the second item (color) that was associated to the same cue, contralateral to the target item. The results demonstrate that alpha/beta power decreases over the hemisphere where the target item is stored (Figure 5C), replicating the basic pattern of Khader and Rösler (2011). More interestingly, however, alpha/beta power also significantly increased over the hemisphere were the competing item was stored (Figure 5C). These results demonstrate that the memory system not only down-regulates alpha/beta synchrony to promote sensory reactivation of a target memory, but also that alpha/beta synchrony can be actively up-regulated, to prevent interference from competing memories. These results support cognitive models of selective memory retrieval suggesting that interfering memory traces are directly inhibited during selective retrieval of target memories (Anderson et al., 1994). As we will demonstrate below (Section "Neural Desynchronization, Memory and Information Theory"), an increase in alpha/beta synchrony reflects a low degree of information processing, thus an increase of alpha/beta power in neural assemblies storing the interfering item can prevent the re-emergence of an unwanted memory trace.

The above cited studies demonstrate that alpha and beta power decreases reflect the successful retrieval of long-term memories. Thereby, alpha and beta power decreases seem to specifically index the reactivation of the sensory features of a memory trace (Burgess and Gruzelier, 2000; Khader and Rösler, 2011; Waldhauser et al., 2012). However, in contrast to the studies investigating the role of alpha and beta power decreases during memory encoding, much less data seems yet to be available regarding alpha and beta power during memory retrieval. Although retrieval related decreases in alpha power have been observed in the hippocampus (Staresina, personal communication), there are no published studies yet examining alpha and beta power decreases with intracranial EEG or simultaneous EEG-fMRI recordings. Therefore, little is known about the brain regions generating these power decreases during retrieval. If alpha and beta power decreases truly reflect the sensory reactivation of a memory trace, they should occur in sensory specific cortical regions (e.g., fusiform gyrus for faces) and also memory specific brain regions (e.g., hippocampus).

\section{NEUROPHYSIOLOGICAL CONSIDERATIONS OF ALPHA/BETA POWER DECREASES}

In order to understand how power decreases that are measured with EEG/MEG on a macroscopic level and intracranial EEG affect neural processing, one needs to consider the interaction between the local field potential and the firing of the single neurons. The neurophysiological model of EEG/MEG signal generation assumes that the local field potential reflects the summated

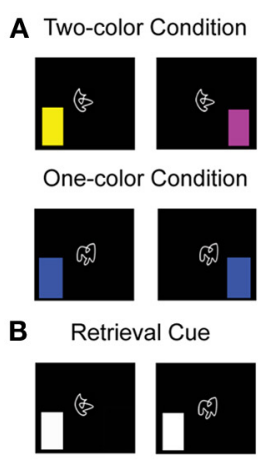

FIGURE 5 | Alpha/beta power during selective retrieval. (A) Subjects encoded shape-color pairs. In one condition two colors were associated with a shape, in the other condition one color was associated with a shape. (B) During the selective retrieval phase the shape was presented together with a cue, specifying the to-be-retrieved target color. (C) Alpha/beta power

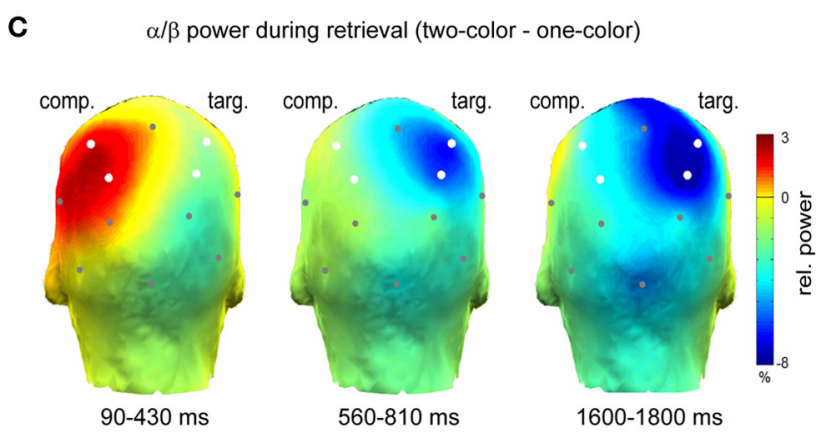

(11.5-20 Hz) during selective retrieval is shown. The color bar denotes the difference in relative power (\%) between the two-color and the one-color condition. Note the increase in alpha/beta power over the competitor hemisphere (comp) and the decrease in power over the target hemisphere (targ). Figure adapted from Waldhauser et al. (2012). 
input of thousands of postsynaptic potentials (inhibitory or excitatory) to an underlying neural assembly (Hämäläinen et al., 1993). Therefore, highly synchronized neural input gives rise to high deflections in the local field potential, and thus increases the power in a specific frequency band (Pfurtscheller and Aranibar, 1977). It has been demonstrated that such synchronized activity induces synchronized firing of the underlying neurons. This is corroborated by studies in primates and humans, showing that neurons fire phase-locked to the local field potential (Lee et al., 2005; Jacobs et al., 2007; Haegens et al., 2011). We can thus infer, that oscillatory power in the EEG reflects the degree of synchronized firing of local cell assemblies. Before we can relate the decreases in local neural synchrony to memory relevant processes, an important alternative explanation needs to be considered.

Several prior studies noticed that power decreases in the alpha and beta frequency bands are paralleled with concurrent power increases in the gamma frequency range $(>40 \mathrm{~Hz})$. This inverse relationship was mostly observed in studies employing visual attention paradigms in primates and humans. For instance, Fries et al. (2001) demonstrated that attention to a stimulus enhances gamma power in the receptive field in V4, while decreasing power in the alpha/beta frequency range. Similar effects were observed in humans in the MEG (Wyart and Tallon-Baudry, 2008). Kilner et al. (2005) acknowledged this negative relationship and suggested that it reflects a frequency shift of oscillatory activity from lower to higher frequencies, which might generally occur during active cortical processing. If this is true, power decreases in alpha/beta frequency should inevitably be accompanied by gamma power increases. However, this was not the case in the two subsequent memory studies from our laboratory; the decreases in alpha/beta power either occurred in a completely different time window than the increases in gamma power (gamma: 0-500 ms vs. alpha/beta: 500-1500 ms; Hanslmayr et al., 2009a), or no effects in the gamma frequency range were observed at all (Hanslmayr et al., 2011b). However, one could still argue that the increases in gamma power might be too local, and might thus be too weak, to be detected with scalp EEG. This interpretation can be ruled out by intracranial electrophysiological studies in primates, showing that selective attention can either decrease or increase gamma power depending on the cortical site, independently of the decreases in alpha/beta power (Chalk et al., 2010; Khayat et al., 2010). Moreover, a recent combined EEGfMRI study demonstrated that the decreases in alpha/beta power are not correlated on a trial-by-trial basis with the increases in gamma power (Scheeringa et al., 2011). Taken together, these results suggest that there is no simple inverse relationship between gamma power increases and alpha/beta power decreases. Instead, the results are more consistent with the view that the alpha/beta and gamma frequencies act independently from each other, thus ruling out the idea of alpha/beta power decreases being an epiphenomenon of power increases in higher frequencies.

\section{NEURAL DESYNCHRONIZATION, MEMORY AND INFORMATION THEORY}

Having established that power decreases in the alpha and beta frequency band, presumably reflecting a desynchronization of local neural assemblies, play an important role for long-term memory, the crucial question is how such desynchronization could possibly aid encoding and retrieval of information in episodic memory. Intuitively, one might think that information is encoded in synchronized rather than desynchronized firing rates, which renders the results described earlier quite puzzling. However, from mathematical models of information theory it can be derived that synchronization is disadvantageous for storing information, as it reduces the richness of information. Entropy, a measure of the richness of information encoded in a sequence of events, can be quantified as the likelihood to which a specific event is expected (Shannon and Weaver, 1949). The underlying idea is that the more an event can be predicted, the less information it carries, or in other words, the more redundant this event is. This is typically illustrated with a coin tossing game. If one tosses a coin, there are two equally probable outcomes and each new toss will give new information, as each toss is independent from the previous coin tosses. Therefore, the information that is obtained in such a coin tossing game is 1 bit per toss, as calculated with Shannon's Entropy. If the coin would be faked such that each toss produces the same outcome, Shannon's Entropy would be zero, indicating minimal degree of information.

Applying this concept to the firing of neurons, it becomes clear that there is an inverse relationship between the richness of information that is encoded in the firing rate of a neural assembly and the synchrony of these firing patterns. This is illustrated in a simple simulation example, depicted in Figure 6. In this simulation, the firing rates of a local neural assembly $(N=50)$ were simulated with no synchronization (Figure 6A, left), a low degree of synchronization (Figure 6A, middle), and a very high degree of synchronization (Figure 6A, right). The sum of spikes is the same across the three conditions (approx. 450). Presumably, the varying degrees of synchronization would be reflected in the power-spectrum of the EEG, with increasing power reflecting increased synchrony (Figure 6B). Applying information theory measures, such as Shannon's Entropy, to the three different conditions, reveals that the degree of information that is encoded in the spiking patterns, increases as a function of desynchronization, with the richest information being encoded in the most desynchronized firing pattern (Figure 6C). This can also be seen in the power of the frequency spectrum, where power correlates negatively with Shannon's Entropy (Figure 6D). This simulation illustrates that the more information needs to be encoded, the more desynchronized the firing of local neural assemblies needs to be. We hypothesize that such desynchronization demands are reflected by the relative decreases in alpha/beta power.

This view is in line with a huge amount of literature in animal research and computational neuroscience applying information theory to analyze how populations of neurons encode sensory stimuli (Barlow, 1961) and how the brain reads out this information (Bialek et al., 1991). These studies reveal that synchronized firing of neurons can be treated as redundant, whereas desynchronized firing patterns reflect efficient information encoding. The idea is nicely demonstrated in a recent study by Schneidman et al. (2011), who showed that neurons that fire synchronously with other neurons convey very little amount of information on downstream neurons, whereas neurons that fire at times where other neurons are silent convey much more information. Therefore, 


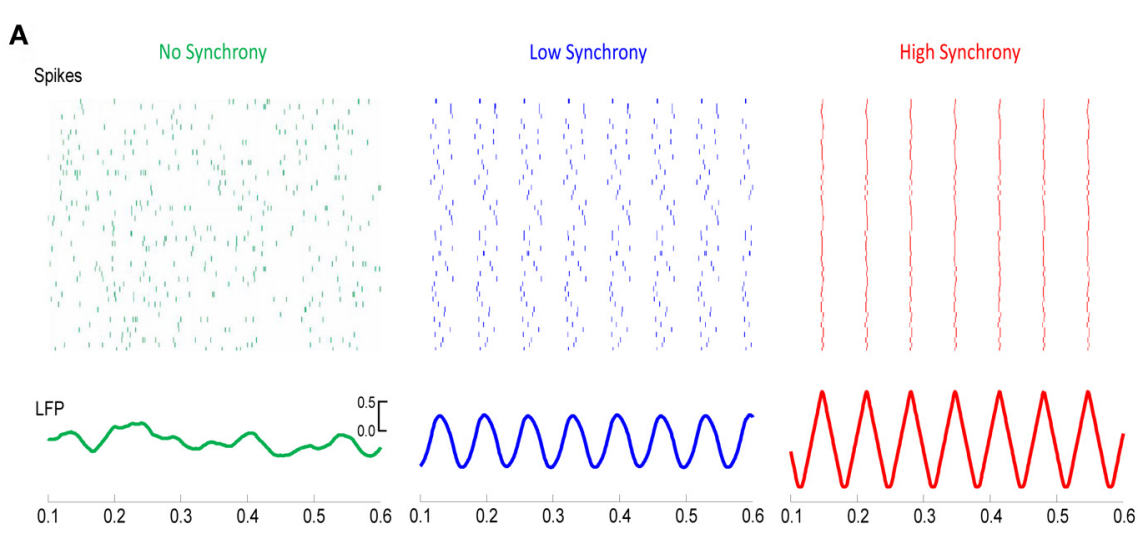

B

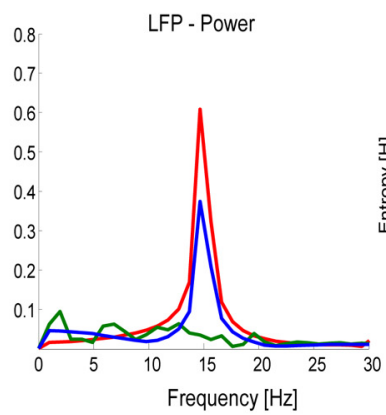

C

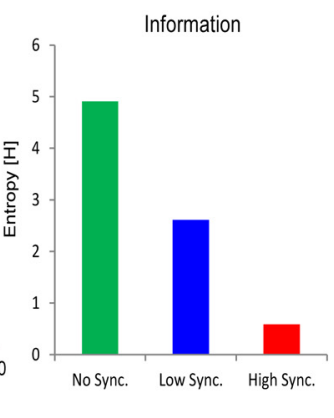

D

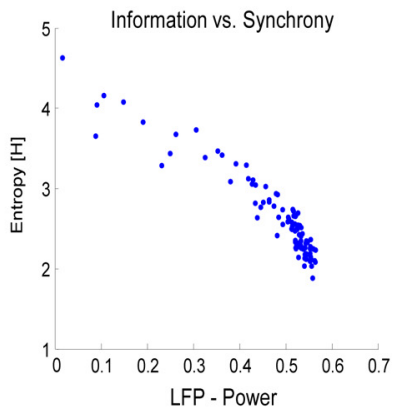

FIGURE 6 | The link between desynchronization and information. (A) Firing rates for a population of neurons $(N=50)$ was simulated with either no synchrony (left panel), a low degree of synchrony (middle panel), or a high degree of synchrony (right panel). The total number of spikes in each population was the same. The lower panels plot the corresponding local field potentials (LFP). (B) The power $(15 \mathrm{~Hz})$ in the LFP increases as a function of synchrony. (C) Information, calculated with Shannon's Entropy, derived from the firing rates of the three neural populations is plotted. (D) The relation between LFP power and information is plotted for simulations with varying degrees of synchrony. Note the inverse relationship between information and synchrony/ LFP-power. desynchronization can be treated as a prerequisite for the emergence of a synergistic population code, in which each neuron represents a unique feature of the encoded information. This idea is also underlined by several animal studies in the visual attention domain, showing that attention decreases synchronization in the primary visual cortex (Chalk et al., 2010), and that the reliability of information that is represented in a cortical area increases as a function of desynchronized firing patterns (Goard and Dan, 2009). Moreover, these ideas are in line with clinical studies showing that patients with memory problems (Alzheimer's disease, mild cognitive impairment) show less stimulus-induced beta power decreases (e.g., Kurimoto et al., 2012).

To relate these desynchronization effects to long-term memory, it is important to consider what the advantage of such a mechanism would be for the long-term memory system, and for the episodic memory system in particular. How can neural desynchronization be related to the cellular substrates of episodic memory formation (e.g., long-term potentiation)? One hallmark of episodic memory is the uniqueness of the episodes that are stored in this particular memory system (Tulving, 1972). Therefore, the neural substrate of this system has to create a unique code for each episode stored in the memory system. Given the vast amount of events that we experience in the course of our life, the creation of unique codes for each episode represents a viable problem for any theoretical memory model, which is known as the problem of pattern separation (Marr, 1971; Alvarez and Squire, 1994; Norman, 2010). Desynchronization might be a quite useful solution to this problem, because an infinite number of unique firing patterns can be generated, even in a very small network of neurons. As illustrated by Schneidman et al. (2011), desynchronized firing could also lead to LTP depending on the underlying neural architecture. This idea is illustrated in Figure 7. If two pyramidal neurons, $\mathrm{P} 1$ and $\mathrm{P} 2$, project directly on a downstream neuron (D) via excitatory synapses, synchronized firing between P1 and P2 enhances the likelihood that the down-stream neuron also fires, thus inducing LTP (Figure 7A). However, if one neuron (P1) has direct excitatory projections, and another neuron (P2) has indirect inhibitory projections to a down-stream neuron, synchronous firing would not cause a discharge of the down-stream neuron because the excitatory input from neuron P1 is blocked by the inhibitory input from neuron P2 (Figure 7B). In such a case, only desynchronized firing would enable the down-stream neuron to selectively listen to the information conveyed by a specific neuron, and also enhances the chance that the 
A

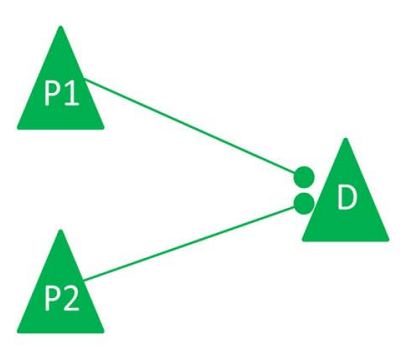

P1

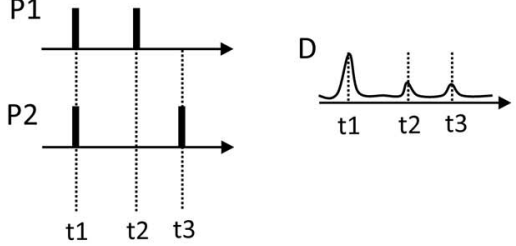

FIGURE 7 | Two different neural connection properties and their relation to synchrony and LTP are shown. (A) Two pyramidal neurons (P1 and P2) have excitatory projections to a down-stream neuron $\mathrm{D}$. If these neurons fire in synchrony (as it happens at $\mathrm{t} 1$ ), their impact on the down-stream neuron adds up, thus increasing the likelihood of a discharge and LTP. If they fire asynchronously (t2 and t3) there is low probability that $\mathrm{D}$ will discharge

\section{B}
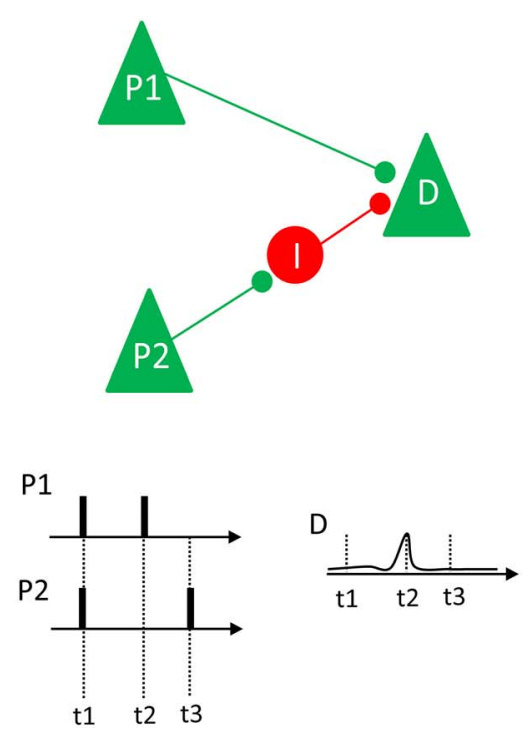

and show LTP. (B) Neuron P1 has excitatory projections to the downstream neuron $D$, whereas neuron P2 has an indirect inhibitory projection to D, via an inhibitory interneuron I. In this case, synchronized firing (at t1) does not add up. Instead, only desynchronized firing (at t2) would lead to a depolarization of D, enhancing the likelihood of LTP. Figure inspired by Schneidman et al. (2011). down-stream neuron discharges in response to this input, thus inducing LTP.

\section{RELATION TO OTHER THEORIES}

As noted above, there is a vast body of computational neuroscience literature applying entropy measures to investigate how the brain reads and codes sensory information (Bialek et al., 1991; Barlow, 2001; Schneidman et al., 2011). Inspired by these studies, we applied some of the basic principles to provide a mechanistic explanation for the role of alpha and beta power decreases for human long-term memory formation and retrieval. These principles, however, do not exclusively apply to memory processes nor are they restricted to the alpha and beta frequency range. In fact, they can generally be applied to other cognitive domains (e.g., attention; Goard and Dan, 2009) and other frequency ranges (e.g., gamma power decreases; Chalk et al., 2010). Future studies, which do not only focus on neural synchronization but also on neural desynchronization, should integrate these concepts to reveal the usefulness of information theory principles for cognitive neuroscience.

Entropy measures have recently also been applied to construct a global brain model, viewing the brain as a prediction making machine trying to reduce its energy costs by keeping its entropy low (Friston, 2010). Similar concepts can also be found in a recent memory model, aiming to explain the memorability of predictable and unpredictable events (Henson and Gagnepain, 2010). We stress to point out, however, that Entropy, as it is used in this paper, has no relation to these concepts. In our model, Entropy refers to the complexity (i.e., predictability) of neural spike patterns and the information that is encoded in these spiking patterns, and not to the predictability of stimuli or memory events.

A recent framework postulated that alpha amplitude modulations regulate the inhibitory level of the cortex (Klimesch et al., 2007; Jensen and Mazaheri, 2010). Thereby, increases in alpha amplitudes reflect inhibition of task irrelevant brain regions and a growing body of literature provides evidence for this assumption (see Klimesch et al., 2007; Jensen and Mazaheri, 2010; for reviews). The here proposed framework is generally in line with this hypothesis in suggesting that decreases in alpha/beta oscillations reflect active information processes. However, the present framework goes one step further, in linking decreases of local alpha/beta synchronization to information theory measures, thus providing a mechanistic explanation of these power decreases.

In opposition to the inhibitory view of alpha oscillations, Palva and Palva $(2007,2011)$ postulated an active processing view, suggesting that alpha oscillations regulate information processing in task relevant brain regions, with a strong emphasis on working memory. In their studies, Palva and Palva focus strongly on inter-areal phase synchrony and show a monotonic increase in alpha and beta phase synchrony with memory load between frontal and parietal areas (e.g., Palva et al., 2010). Such increased synchrony seems to be at odds with our ideas of synchrony being inversely related to information processing. However, this discrepancy can be resolved when we distinguish between large scale synchrony, as measured by inter-areal phase synchronization, and local synchrony, as measured by amplitude. Our hypothesis was derived from memory studies only investigating amplitude modulations and thus cannot be extended to 
large scale synchronization. In fact, there might even be an inverse relationship between local and global synchronization, such that a decrease in local synchrony correlates with an increase in large scale synchrony (e.g., Freunberger et al., 2009).

\section{CONCLUDING REMARKS, TESTABLE PREDICTIONS, AND OPEN QUESTIONS}

In the current paper we reviewed studies showing that desynchronized neural activity, as reflected by power decreases in alpha and beta frequencies, is beneficial for memory processes. The main purpose of this paper is to offer a first, plausible mechanistic explanation for the role of alpha and beta power decreases, which are robustly observed and, therefore, call for an explanation. Applying information theory to the degree of synchrony in neural firing patterns, we showed that information is inversely related to synchrony. Therefore, the degree of desynchronization in the alpha and beta power ranges might represent the richness of information encoded in a memory trace. From this idea, several testable predictions can be derived. For instance, externally inducing synchrony in task relevant brain regions, e.g., via repetitive transcranial magnetic stimulation (rTMS) or transcranial AC stimulation (tACS) during encoding and retrieval, should impair memory performance. Indeed, previous studies showed that rTMS in the alpha and beta frequency range delivered at the left dorso-lateral PFC impairs memory encoding (Innocenti et al., 2010; Rossi et al., 2011). However, future studies should also investigate if these effects extend to the left inferior PFC and whether they are specific to the alpha/beta range, or whether externally induced synchrony generally impairs memory performance, independent of the stimulation frequency. Another prediction that follows is that the degree of alpha and beta synchronization is inversely related to the amount of information

\section{REFERENCES}

Addante, R. J., Watrous, A. J., Yonelinas, A. P., Ekstrom, A. D., and Ranganath, C. (2011). Prestimulus theta activity predicts correct source memory retrieval. Proc. Natl. Acad. Sci. U.S.A. 108, 10702-10707.

Alvarez, P., and Squire, L. R. (1994). Memory consolidation and the medial temporal lobe: a simple network model. Proc. Natl. Acad. Sci. U.S.A. 91, 7041-7045.

Anderson, M. C., Bjork, R. A., and Bjork, E. L. (1994). Remembering can cause forgetting: retrieval dynamics in long-term memory. $J$. Exp. Psychol. Learn. Mem. Cogn. 20, 1063-1087.

Anderson, M. C., and Green, C. (2001). Suppressing unwanted memories by executive control. Nature 410, 366-369.

Axmacher, N., Mormann, F., Fernández, G., Elger, C. E., and Fell, J. (2006). Memory formation by neuronal synchronization. Brain Res. Rev. 52, 170-182.
Barlow, H. (2001). Redundancy reduction revisited. Comput. Neural. Syst. 12, 241-253.

Barlow, H. B. (1961). "The coding of sensory messages," in Current Problems in Animal Behaviour, eds W. H. Thorpe and O. L. Zangwill (Cambridge, UK: Cambridge University Press), 331-360.

Bialek, W., Rieke, F., de Ruyter van Steveninck, R. R., and Warland, D. (1991). Reading a neural code. Science 252, 1854-1857.

Burgess, A. P., and Gruzelier, J. H. (2000). Short duration power changes in the EEG during recognition memory for words and faces. Psychophysiology 37, 596-606.

Buzsáki, G., and Draguhn, A. (2004). Neuronal oscillations in cortical networks. Science 304, 1926-1929.

Chalk, M., Herrero, J. L., Gieselmann, M. A., Delicato, L. S., Gotthardt, S., and Thiele, A. (2010). Attention reduces stimulus-driven gamma frequency oscillations and spike field coherence in V1. Neuron 66, 114-125.

that is retrieved, and the studies by Khader and Rösler (2011) and Waldhauser et al. (2012) confirm this prediction. However, it would be interesting to see whether alpha and beta synchronization can be increased on a voluntary basis to suppress the reactivation of unwanted memories (Anderson and Green, 2001; Hanslmayr et al., 2009b).

The presented framework also raises some important questions. For example, if information is indeed encoded in a desynchronized, rather than a synchronized firing pattern, what is the role that is played by the memory related increases in synchronization that typically occur in the gamma and theta frequency range? If one views synchrony as a continuum, there would be completely chaotic firing on one side and complete synchronization on the other side, none of which is favorable for cognitive processes. In the case of complete chaos, that is total desynchronization, the system would be highly unstable and highly susceptible to information loss due to its lack of redundancy. On the other side, total synchronization, as it typically occurs during states where there is little cognitive processing (e.g., epileptic seizures or slow wave sleep), does not allow variability in the neural code which is required to represent information. In the end, it might turn out that the synchronization and desynchronization effects in different frequency bands reflect an optimal balance between chaotic and systematic neural activity, both of which are necessary ingredients for a functioning memory system.

\section{ACKNOWLEDGMENTS}

The research presented in this work was supported by a grant from the Deutsche Forschungsgemeinschaft (Project HA 5622/11) awarded to Simon Hanslmayr. We thank Franz Huber, Andreas Karrenbauer, and Maria Wimber for helpful discussions in preparing this manuscript.

Craik, F. I. M. (2002). Levels of processing: past, present and future? Memory 10, 305-318.

Dujardin, K., Bourriez, J. L., and Guieu, J. D. (1994). Event-related desynchronization (ERD) patterns during verbal memory tasks: effect of age. Int. J. Psychophysiol. 16, 17-27.

Düzel, E., Habib, R., Schott, B., Schoenfeld, A., Lobaugh, N., McIntosh, A. R., Scholz, M., and Heinze, H. J. (2003). A multivariate, spatiotemporal analysis of electromagnetic time-frequency data of recognition memory. Neuroimage 18, 185-197.

Düzel, E., Penny, W. D., and Burgess, N. (2010). Brain oscillations and memory. Curr. Opin. Neurobiol. 20, 143-149.

Fell, J., and Axmacher, N. (2011) The role of phase synchronization in memory processes. Nat. Rev. Neurosci. 12, 105-118.

Fell, J., Klaver, P., Lehnertz, K., Grunwald, T., Schaller, C., Elger, C. E., and Fernández, G. (2001). Human memory formation is accompanied by rhinalhippocampal coupling and decoupling. Nat. Neurosci. 4, 1259-1264. Fell, J., Ludowig, E., Staresina, B. P., Wagner, T., Kranz, T., Elger, C. E., and Axmacher, N. (2011). Medial temporal theta/alpha power enhancement precedes successful memory encoding: evidence based on intracranial EEG. J. Neurosci. 31, 5392-5397.

Freunberger, R., Fellinger, R., Sauseng, P., Gruber, W., and Klimesch, W. (2009). Dissociation between phaselocked and nonphase-locked alpha oscillations in a working memory task. Hum. Brain Mapp. 30, 3417-3425.

Fries, P., Reynolds, J. H., Rorie, A. E., and Desimone, R. (2001). Modulation of oscillatory neuronal synchronization by selective visual attention. Science 291, 1560-1563.

Friston, K. (2010). The free-energy principle: a unified brain theory? Nat. Rev. Neurosci. 11, 127-138.

Gabrieli, J. D., Poldrack, R. A., and Desmond, J. E. (1998). The role of 
left prefrontal cortex in language and memory. Proc. Natl. Acad. Sci. U.S.A. 95, 906-913.

Goard, M., and Dan, Y. (2009). Basal forebrain activation enhances cortical coding of natural scenes. Nat. Neurosci. 12, 1444-1449.

Gratton, G., Corballis, P. M., and Jain, S. (1997). Hemispheric organization of visual memories. J. Cogn. Neurosci. 9, 92-104.

Guderian, S., Schott, B. H., RichardsonKlavehn, A., and Düzel, E. (2009). Medial temporal theta state before an event predicts episodic encoding success in humans. Proc. Natl. Acad. Sci. U.S.A. 106, 5365-5370.

Haegens, S., Nácher, V., Luna, R., Romo, R., and Jensen, O. (2011). $\alpha$-Oscillations in the monkey sensorimotor network influence discrimination performance by rhythmical inhibition of neuronal spiking. Proc. Natl. Acad. Sci. U.S.A. 108, 19377-19382.

Hämäläinen, M., Hari, R., Illmoniemi, R. J., Knuutila, J., and Lounasmaa, O. V. (1993). Magnetoencephalography_theory, instrumentation, and applications to noninvasive studies of the working human brain. Rev. Mod. Phys. $65,413-497$.

Hanslmayr, S., Gross, J., Klimesch, W., and Shapiro, K. L. (2011a). The role of alpha oscillations in temporal attention. Brain Res. Rev. 67, 331-343.

Hanslmayr, S., Leipold, P., Passtötter, B., and Bäuml, K.-H. (2009b). Anticipatory signatures during voluntary memory suppression. $J$. Neurosci. 29, 2742-2747.

Hanslmayr, S., Spitzer, B., and Bäuml, K.-H. (2009a). Brain oscillations dissociate between semantic and nonsemantic encoding of episodic memories. Cereb. Cortex 19, 1631-1640.

Hanslmayr, S., Staudigl, T., Aslan, A., and Bäuml, K.-H. (2010). Theta oscillations predict the detrimental effects of memory retrieval. Cogn. Affect. Behav. Neurosci. 10, 329-338.

Hanslmayr, S., Volberg, G., Wimber, M., Raabe, M., Greenlee, M. W., and Bäuml, K.-H. T. (2011b). The relationship between brain oscillations and BOLD signal during memory formation: a combined EEG-fMRI study. J. Neurosci. 31, 15674-15680.

Hebb, D. O. (2002). The Organization of Behavior: A Neuropsychological Theory. Mahwah, NJ: Erlbaum.

Henson, R. N., and Gagnepain, P. (2010). Predictive, interactive multiple memory systems. Hippocampus 20, 1315-1326.
Innocenti, I., Giovanelli, F., Cincotta, M., Feurra, M., Polizzotto, N. R., Bianco, G., Cappa, S. F., and Rossi, S. (2010). Event-related rTMS at encoding affects differently deep and shallow memory traces. Neuroimage 53, 325-330.

Jacobs, J., Kahana, M. J., Ekstrom, A. D., and Fried, I. (2007). Brain oscillations control timing of single-neuron activity in humans. $J$. Neurosci. 27, 3839-3844.

Jensen, O., Gelfand, J., Kounios, J., and Lisman, J. E. (2002). Oscillations in the alpha band $(9-12 \mathrm{~Hz})$ increase with memory load during retention in a short-term memory task. Cereb. Cortex 12, 877-882.

Jensen, O., and Mazaheri, A. (2010). Shaping functional architecture by oscillatory alpha activity: gating by inhibition. Front. Hum. Neurosci. 4:186. doi: 10.3389/fnhum.2010.00186

Khader, P. H., Jost, K., Ranganath, C., and Rösler, F. (2010). Theta and alpha oscillations during workingmemory maintenance predict successful long-term memory encoding. Neurosci. Lett. 468, 339-343.

Khader, P. H., and Rösler, F. (2011). EEG power changes reflect distinct mechanisms during long-term memory retrieval. Psychophysiology 48, 362-369.

Khayat, P. S., Niebergall, R., and Martinez-Trujillo, J. C. (2010). Frequency-dependent attentional modulation of local field potential signals in macaque area MT. $J$. Neurosci. 30, 7037-7048.

Kilner, J. M., Mattout, J., Henson, R., and Friston, K. J. (2005). Hemodynamic correlates of EEG: a heuristic. Neuroimage 28, 280-286.

Kim, H. (2011). Neural activity that predicts subsequent memory and forgetting: a meta-analysis of 74 fMRI studies. Neuroimage 54, 2446-2461.

Klimesch, W. (1999). EEG alpha and theta oscillations reflect cognitive and memory performance: a review and analysis. Brain Res. Brain Res. Rev. 29, 169-195.

Klimesch, W., Doppelmayr, M. Schimke, H., and Ripper, B. (1997). Theta synchronization and alpha desynchronization in a memory task. Psychophysiology 34, 169-176.

Klimesch, W., Doppelmayr, M., Stadler, W., Pöllhuber, D., Sauseng, P., and Röhm, D. (2001). Episodic retrieval is reflected by a process specific increase in human electroencephalographic theta activity. Neurosci. Lett. 302, 49-52.

Klimesch, W., Sauseng, P., and Hanslmayr, S. (2007). EEG alpha oscillations: the inhibition-timing hypothesis. Brain Res. Rev. 53 63-88.

Klimesch, W., Schimke, H., Doppelmayr, M., Ripper, B. Schwaiger, J., and Pfurtscheller, G. (1996). Event-related desynchronization (ERD) and the Dm effect: does alpha desynchronization during encoding predict later recal performance? Int. J. Psychophysiol. $24,47-60$.

Kurimoto, R., Ishii, R., Canuet, L., Ikezawa, K., Iwase, M., Azechi M., Aoki, Y., Ikeda, S., Yoshida, T., Takahashi, H., Nakahachi, T., Kazui, H., and Takeda, M. (2012). Induced oscillatory responses during the Sternberg's visual memory task in patients with Alzheimer's disease and mild cognitive impairment. Neuroimage 59, 4132-4140.

Laufs, H., Krakow, K., Sterzer, P., Eger, E., Beyerle, A., Salek-Haddadi, A., and Kleinschmidt, A. (2003) Electroencephalographic signatures of attentional and cognitive default modes in spontaneous brain activity fluctuations at rest. Proc. Natl. Acad. Sci. U.S.A. 100 11053-11058.

Lee, H., Simpson, G. V., Logothetis, N. K., and Rainer, G. (2005). Phase locking of single neuron activity to theta oscillations during working memory in monkey extrastriate visual cortex. Neuron 45, 147-156.

Mantini, D., Perrucci, M. G., Del Gratta, C., Romani, G. L., and Corbetta, M. (2007). Electrophysiological signatures of resting state networks in the human brain. Proc. Natl. Acad. Sci. U.S.A 104, 13170-13175.

Markram, H., Lübke, J., Frotscher, M., and Sakmann, B. (1997). Regulation of synaptic efficacy by coincidence of postsynaptic APs and EPSPs. Science 275, 213-215.

Marr, D. (1971). Simple memory: a theory for archicortex. Philos. Trans. R. Soc. Lond. B Biol. Sci. 262, 23-81.

Meeuwissen, E. B., Takashima, A., Fernández, G., and Jensen, O (2011a). Increase in posterior alpha activity during rehearsal predicts successful long-term memory formation of word sequences. Hum Brain Mapp. 32, 2045-2053.

Meeuwissen, E. B., Takashima, A. Fernández, G., and Jensen, O. (2011b). Evidence for human fronto-central gamma activity during long-term memory encoding of word sequences. PLoS One 6:e21356. doi: 10.1371/journal. pone. 0021356
Moosmann, M., Ritter, P., Krastel, I., Brink, A., Thees, S., Blankenburg, F., Taskin, B., Obrig, H., and Villringer, A. (2003). Correlates of alpha rhythm in functional magnetic resonance imaging and near infrared spectroscopy. Neuroimage 20, 145-158.

Norman, K. A. (2010). How hippocampus and cortex contribute to recognition memory: revisiting the complementary learning systems model. Hippocampus 20, 1217-1227.

Nyhus, E., and Curran, T. (2010). Functional role of gamma and theta oscillations in episodic memory. Neurosci. Biobehav. Rev. 34 1023-1035.

Otten, L. J., and Rugg, M. D. (2001). Task-dependency of the neural correlates of episodic encoding as measured by fMRI. Cereb. Cortex 11, $1150-1160$.

Paller, K. A., and Wagner, A. D. (2002). Observing the transformation of experience into memory. Trends Cogn. Sci. 6, 93-102.

Palva, J. M., Monto, S., Kulashekhar, S., and Palva, S. (2010). Neuronal synchrony reveals working memory networks and predicts individual memory capacity. Proc. Natl. Acad. Sci. U.S.A. 107, 7580-7585.

Palva, S., and Palva, J. M. (2007). New vistas for alpha-frequency band oscillations. Trends Neurosci. 30, 150-158.

Palva, S., and Palva, J. M. (2011). Functional roles of alpha-band phase synchronization in local and large-scale cortical networks. Front. Psychol. 2:204. doi: 10.3389/fpsyg.2011.00204

Pfurtscheller, G., and Aranibar, A. (1977). Event-related cortical desynchronization detected by power measurements of scalp EEG. Electroencephalogr. Clin. Neurophysiol. 42, 817-826.

Pfurtscheller, G., Neuper, C., Andrew, C., and Edlinger, G. (1997). Foot and hand area mu rhythms. Int. J. Psychophysiol. 26, 121-135.

Rossi, S., Innocenti, I., Polozzotto, N. R., Feurra, M., De Capua, A., Ulivelli, M., Bartalini, S., and Cappa, S. F. (2011). Temporal dynamics of memory trace formation in the human prefrontal cortex. Cereb. Cortex 21, 368-373.

Scheeringa, R., Fries, P., Petersson, K.-M., Oostenveld, R., Grothe, I. Norris, D. G., Hagoort, P., and Bastiaansen, M. C. M. (2011). Neuronal dynamics underlying high- and low-frequency EEG oscillations contribute independently to the human BOLD signal. Neuron $69,572-583$. 
Schneidman, E., Puchalla, J. L., Segev, R., Harris, R. A., Bialek, W., and Berry, M. J. (2011). Synergy from silence in a combinatorial neural code. J. Neurosci. 31, 15732-15741.

Sederberg, P. B., Gauthier, L. V., Terushkin, V., Miller, J. F., Barnathan, J. A., and Kahana, M. J. (2006). Oscillatory correlates of the primacy effect in episodic memory. Neuroimage 32, 1422-1431.

Sederberg, P. B., Kahana, M. J., Howard, M. W., Donner, E. J., and Madsen, J. R. (2003). Theta and gamma oscillations during encoding predict subsequent recall. J. Neurosci. 23, 10809-10814.

Sederberg, P. B., Schulze-Bonhage, A., Madsen, J. R., Bromfield, E. B. McCarthy, D. C., Brandt, A., Tully, M. S., and Kahana, M. J. (2007). Hippocampal and neocortical gamma oscillations predict memory formation in humans. Cereb. Cortex 17, 1190-1196.

Shannon, C. E., and Weaver, W. (1949). The Mathematical Theory of Communication. Urbana, IL: University of Illinois Press.

Spitzer, B., Hanslmayr, S., Opitz, B., Mecklinger, A., and Bäuml, K.-H. (2009). Oscillatory correlates of retrieval-induced forgetting in recognition memory. J. Cogn. Neurosci. 21, 976-990.

Staudigl, T., Hanslmayr, S., and Bäuml, K.-H. T. (2010). Theta oscillations reflect the dynamics of interference in episodic memory retrieval. $J$. Neurosci. 30, 11356-11362.

Tulving, E. (1972). "Episodic and semantic memory," in Organization of Memory, eds E. Tulving and W. Donaldson (New York, NY: Academic Press, Inc.), 382-402.

Tulving, E., and Thomson, D. M. (1973). Encoding specificity and retrieval processes in episodic memory. Psych. Rev. 80, 352-373.

Waldhauser, G., Johansson, M., and Hanslmayr, S. (2012). Brain oscillations indicate inhibition of interfering visual memories. J. Neurosci. 32, 1953-1961.

Weiss, S., and Rappelsberger, P. (2000). Long-range EEG synchronization during word encoding correlates with successful memory performance. Brain Res. Cogn. Brain Res. 9, 299-312.

Wyart, V., and Tallon-Baudry, C. (2008). Neural dissociation between visual awareness and spatial attention. J. Neurosci. 28, 2667-2679.

Zion-Golumbic, E., Kutas, M., and Bentin, S. (2010). Neural dynamics associated with semantic and episodic memory for faces: evidence from multiple frequency bands. $J$. Cogn. Neurosci. 22, 263-277.
Conflict of Interest Statement: The authors declare that the research was conducted in the absence of any commercial or financial relationships that could be construed as a potential conflict of interest.

Received: 15 December 2011; accepted: 16 March 2012; published online: 03 April 2012

Citation: Hanslmayr S, Staudigl T and Fellner M-C (2012) Oscillatory power decreases and long-term memory: the information via desynchronization hypothesis. Front. Hum. Neurosci. 6:74. doi: 10.3389/fnhum.2012.00074

Copyright (c) 2012 Hanslmayr, Staudigl and Fellner. This is an open-access article distributed under the terms of the Creative Commons Attribution Non Commercial License, which permits noncommercial use, distribution, and reproduction in other forums, provided the original authors and source are credited. 